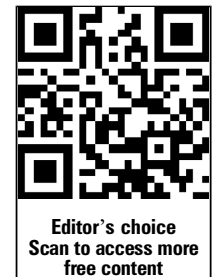

free content
- Additional material is published online only. To view please visit the journal online (http://dx.doi.org/10.1136/ jnnp-2013-306948).

${ }^{1}$ Neuroscience Research Australia, Sydney, Australia ${ }^{2}$ UNSW Medicine, University of New South Wales, Sydney, Australia

${ }^{3} A R C$ Centre of Excellence in Cognition and its Disorders, Sydney, Australia ${ }^{4}$ Disciplines of Pathology, Sydney Medical School, The University of Sydney, Sydney, Australia

${ }^{5}$ Disciplines of Medicine Sydney Medical School, The University of Sydney, Sydney, Australia

Correspondence to: Professor Glenda Halliday, Neuroscience Research Australia, Barker Street, Randwick 2031, Australia; g.halliday@neura.edu.au

Received 10 October 2013 Revised 26 November 2013 Accepted 5 December 2013 Published Online First 13 January 2014

\title{
New criteria for frontotemporal dementia syndromes: clinical and pathological diagnostic implications
}

\author{
Leone Chare, ${ }^{1,2}$ John R Hodges, ${ }^{1,2,3}$ Cristian E Leyton, ${ }^{1,3}$ Ciara McGinley, ${ }^{4}$ \\ Rachel H Tan, ${ }^{1}$ Jillian J Kril, ${ }^{4,5}$ Glenda M Halliday ${ }^{1,2}$
}

\section{ABSTRACT}

Objective To assess the impact of new clinical diagnostic criteria for frontotemporal dementia (FTD) syndromes, including primary progressive aphasias (PPA), on prior clinical diagnosis and to explore

clinicopathological correlations.

Methods 178 consecutive neuropathologically ascertained cases initially diagnosed with a FTD syndrome were collected through specialist programmes: the Cambridge Brain Bank, UK, and Sydney Brain Bank, Australia. 135 cases were reclassified using the revised diagnostic criteria into behavioural variant (bvFTD), semantic variant PPA (sv-PPA), non-fluent/agrammatic variant PPA (nfv-PPA) and logopenic variant PPA (IvPPA). Pathological diagnoses included FTLD-tau, FTLDTDP, FTLD-FUS, FTLD-UPS, FLTD-ni and Alzheimer's disease (AD). Statistical analyses included $\chi^{2}$ tests, analyses of variance and discriminant statistics.

Results Comparison of the original and revised diagnosis revealed no change in $90 \%$ of bvFTD and svPPA cases. By contrast, $51 \%$ of nfv-PPA cases were reclassified as Iv-PPA, with apraxia of speech and sentence repetition assisting in differentiation. Previous patterns of pathology were confirmed, although more AD cases occurred in FTD syndromes (10\% bvFTD, $\sim 15 \%$ sv-PPA and $~ 30 \%$ nfv-PPA) than expected. AD was the dominant pathology (77\%) of Iv-PPA. Discriminant analyses revealed that object agnosia, phonological errors and neuropsychiatric features differentiated AD from FTLD.

Conclusions This study provides pathological validation that the new criteria assist with separating PPA cases with AD pathology into the new Iv-PPA syndrome and found that a number of diagnostic clinical features (disinhibition, food preferences and naming) did not assist in discriminating the different FTD syndromes.

\section{INTRODUCTION}

Frontotemporal dementia (FTD) is clinically and pathologically heterogeneous. The recent International consensus papers recognise four main clinical variants-a behavioural variant (bvFTD) characterised by prominent early personality or behavioural changes ${ }^{1}$ and three primary progressive aphasia (PPA) syndromes ${ }^{2}$ : semantic variant or sv-PPA (previously known as semantic dementia), a non-fluent/agrammatic variant or nfv-PPA (previously known as progressive non-fluent aphasia) and a logopenic variant or lv-PPA. The latter syndrome is distinguished by impairment of lexical retrieval and sentence repetition. ${ }^{2}$ In parallel consensus opinion recognises five major pathological subtypes of frontotemporal lobar degeneration (FTLD) (FTLD-tau，FTLD-TDP, FTLD-FUS， FTLD-UPS and FTLD-no inclusions or FTLD-ni), ${ }^{3}$ although it is also known that a proportion of clinically diagnosed FTD patients have underlying Alzheimer's disease (AD) pathology notably those with lv-PPA. ${ }^{45}$

The recent changes in both clinical and pathological criteria have yet to be independently evaluated and validated in a large autopsy series. Such an evaluation would also inform on the impact on clinical diagnosis of implementing the new criteria. This study assessed the impact of these new criteria ${ }^{12}$ on a cohort of previously clinically diagnosed FTD cases and also assessed which diagnostic features assist most in distinguishing the clinical syndromes. Furthermore, the association between the different FTD syndromes (defined using the new criteria) and underlying pathology was examined, and the combination of features that might better differentiate underlying pathologies, particularly the detection of patients with $\mathrm{AD}$ pathology, examined.

\section{METHODS}

Case ascertainment and selection

Inclusion criteria for this study were participation in multidisciplinary research programmes that longitudinally investigating neurodegenerative dementias in Cambridge and Sydney and an initial clinical diagnosis of a FTD syndrome (bvFTD, semantic dementia and progressive non-fluent aphasia) $)^{6} 7$ and a confirmatory neuropathological diagnosis ${ }^{6-8}$ or a pathological diagnosis of AD. ${ }^{9}$ Patients with coexisting motor impairments were not excluded from the analysis, but a dominant motor disorder was an exclusion criteria. These specialist programmes for FTD syndromes were developed in 1990 to ensure high (90\%) enrolment and success in obtaining brain tissue donations and were approved by the Addenbrooke's Hospital Local Ethics Committee and the Human Ethics Committees of the South Eastern and Illawarra Area Heath Service and the Universities of New South Wales and Sydney. Following the exclusion of three cases due to alternate pathologies (two with dementia with Lewy bodies and one with glioblastoma), 175 consecutive neuropathologically ascertained FTD cases met the inclusion criteria (figure 1). Review of the records revealed that 27 cases had a dominant movement disorder and a further 13 cases had insufficient clinical data, leaving 135 for reclassification (figure 1). 
Initial Case selection

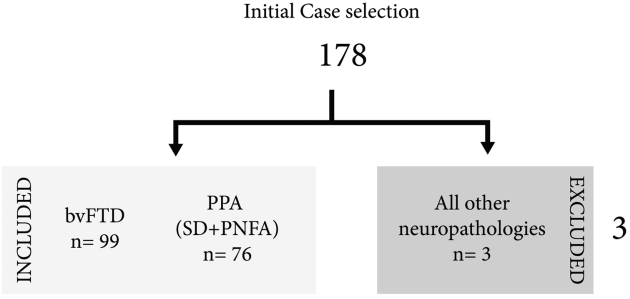

Retrospective application of new diagnostic criteria

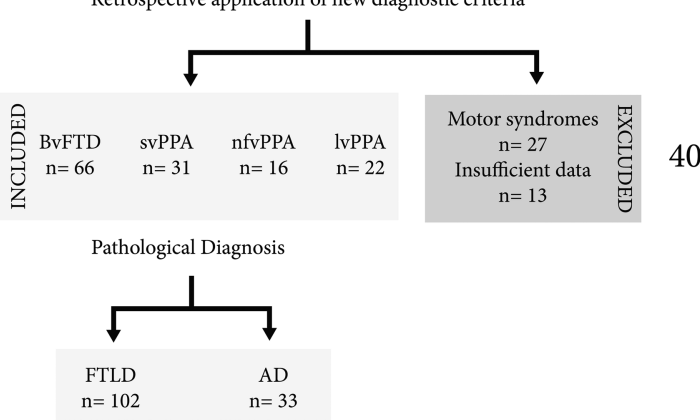

Figure 1 Case ascertainment and procedures.

Revised clinical diagnostic criteria ${ }^{1}{ }^{2}$ were applied retrospectively from symptom onset. Evidence of diagnostic features (see box 1) was endorsed if noted in neurological reports, standardised neuropsychiatric interviews and neuropsychological testing (refer to online supplementary methods). Particular attention was paid to the first clinical assessment and diagnosis, the symptom intensity and the temporal occurrence of features characteristic of one of the clinical variants of FTD, and reclassification was achieved by consensus (LC and JRH). Revised neuropathological classification ${ }^{3}$ of all cases was performed (figure 2).

\section{Box 1 Clinical features extracted from patient files*}

Frontal behavioural featurest: disinhibition, apathy, loss of empathy, stereotyped/perseverative behaviour, alterations in food preferences, executive deficits.

Language features $¥$ : word finding difficulties, apraxia of speech, agrammatism, anomia, impaired single word comprehension, impaired object knowledge, phonologic errors, impaired word/sentence repetition, impaired sentence comprehension, surface dyslexia, dysgraphia.

Motor signs§: parkinsonism, apraxia, gait disturbance, orobuccal apraxia.

Other neuropsychiatric featurest: delusions, hallucinations. Other†१: prosopagnosia, mental rigidity, irritability, aggression. *Features were scored as positive if documented as present and negative if stated as absent or not commented on in files. †Assessed primarily by informant based questionnaires. for example, Cambridge Behavioural Inventory or Neuropsychiatric Inventory.

$¥$ Assessed by standardised clinical language evaluation and neuropsychological testing (refer to online supplementary methods).

$\S$ Assessed by neurological examination.

१From carer report plus questionnaires.
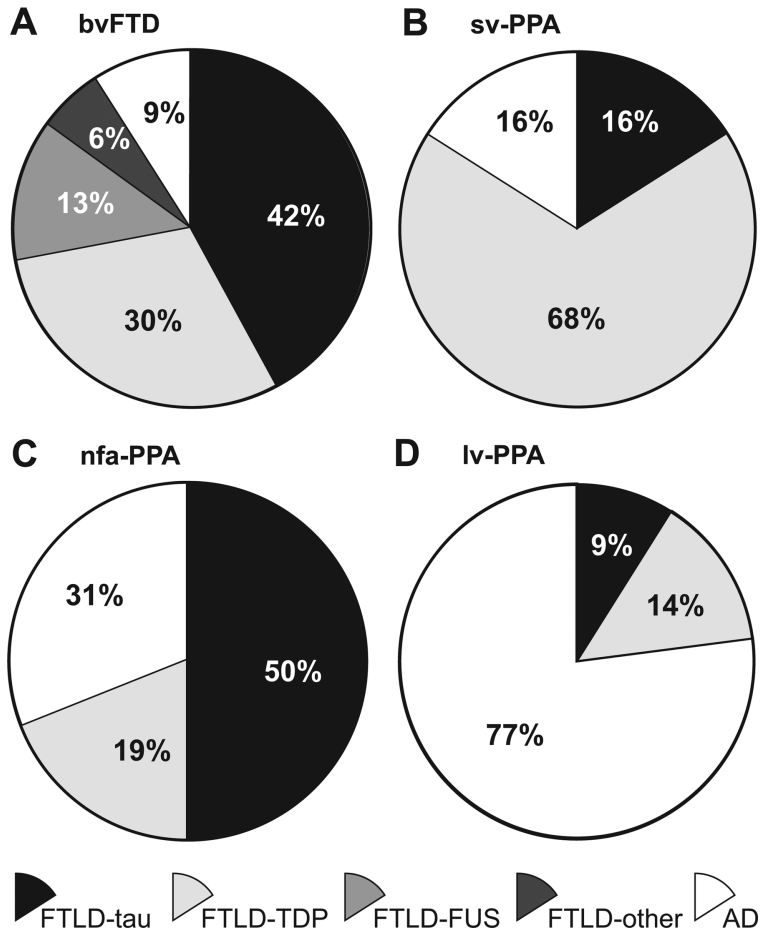

Figure 2 Neuropathologies in clinical frontotemporal dementia syndromes showing the expected variability, although Alzheimer's disease is prominent in non-fluent/agrammatic variant-primary progressive aphasias.

\section{Neuropathological classification using new diagnostic criteria}

While all cases had been consistently classified as having either taupositive or tau-negative pathologies over the collection period, systematic classification into the newly recognised major molecular classes (FTLD-tau, FTLD-TDP, FTLD-FUS, FTLD-UPS and FTLD-ni) ${ }^{3}$ had not been performed. In addition, new diagnostic criteria for AD were recently published. ${ }^{10}$ Revised neuropathological classification of all cases was performed by analysing newly cut frontal and medial temporal lobe sections stained immunohistochemically using standard techniques ${ }^{11}$ for phospho-tau (CloneAT8, Cat\# MN1020, Thermo Scientific Australia, Scoresby, Victoria, 1:1000), phospho-TDP-43 (Cat\# TIP-PTD M01, Cosmo Bio, Tokyo, Japan, 1:80 000), native TDP (Cat\#10782-2-AP, Protein Tech, Chicago, Illinois, 1:1000), FUS (Cat\# HPA008784, Sigma Aldrich Australia, Dural, New South Wales, 1:500), p62 (Cat\#610833, BD Biosciences Australia, North Ryde, New South Wales, 1:250), A $\beta$ (Cat\#SIG39320, Covance, North Ryde, New South Wales, Clone 6E10, 1:500) and $\alpha$-synuclein (Cat\#610787, BD Transduction Labs, San Jose, California, 1:500) and applying the new criteria. Note that modified Bielschowsky silver staining had been consistently performed on all cases for the assessment of neuritic pathologies.

\section{Statistical analyses}

All statistical analyses were performed using SPSS (IBM SPSS statistics V.21; SPSS Inc, Chicago, Illinois, USA). Details of the statistical plan can be found in the supplementary files. Demographic differences were assessed using analyses of variance, differences in the prevalence of syndromes and features using $\chi^{2}$ tests, differences between new and previous clinical diagnoses using $\kappa$ statistics and the diagnostic clinical features differentiating clinical (bvFTD, svPPA, nfv-PPA vs lv-PPA) or 
pathological (either FTLD vs AD or FTLD subtype) diagnoses analysed using discriminate function analysis.

\section{RESULTS}

\section{Characterisation of the clinical cohorts}

As shown in table 1 , there was a male predominance in all clinical subtypes of FTD $(\sim 70 \%)$ and bvFTD had a significantly younger age at death. sv-PPA cases had longer mean disease duration. Additional details by clinical center in supplementary results and supplementary table e1. Discriminative analysis revealed that $12 / 27$ features discriminated the clinical subgroups with 93\% accuracy (Wilks' lambda $=0.024, \mathrm{p}<0.0001$, table 1). For bvFTD, the most discriminating features were a high prevalence of stereotyped behaviours and, unsurprisingly, a relative lack of language and motor features. For sv-PPA, the presence of word finding difficulties (WFD), single word comprehension deficits (SWC), surface dyslexia and prosopagnosia and relative absence of agrammatism, repetition deficits and orobuccal apraxia were the most discriminating features. The most discriminating features for nfv-PPA were the presence of apraxia of speech (AoS) and relatively spared SWC while lv-PPA was best discriminated by the presence of WFD, preserved word comprehension and lack of orobuccal apraxia. Interestingly, both nfv-PPA and lv-PPA had a relative lack of behavioural deficits compared with those with sv-PPA (additional information and results can be found in the supplementary table 1 ).

\section{Impact of new criteria on previous clinical phenotypes}

For bvFTD and sv-PPA, the majority of cases did not change diagnosis. All cases of bvFTD diagnosed using the new criteria were bvFTD using older criteria (table 2). A small number $(7 \%)$ of previously diagnosed bvFTD had sufficient language features at onset, such as anomia, phonological errors and comprehension deficits, to warrant reclassification into one of the three

Table 1 Demographics and the proportion of cases in each group with the main clinical features of the new FTD clinical phenotypes

\begin{tabular}{|c|c|c|c|c|c|}
\hline & bvFTD (N=66) & Sv-PPA (N=31) & $n f v-P P A(N=16)$ & Iv-PPA (N=22) & $p$ Value \\
\hline$\%$ male $/ \%$ female & $65 / 35$ & $56 / 43$ & $65 / 35$ & $86 / 14$ & 0.14 \\
\hline Age at onset (years) & $57(10)$ & $61(7)$ & $65(8)$ & $62(9)$ & 0.004 \\
\hline Age at diagnosis (years) & $61(9)$ & $65(7)$ & $70(8)$ & $66(8)$ & 0.002 \\
\hline Age at death (years) & $66(10)$ & $73(7)$ & $75(7)$ & $71(8)$ & $<0.0001$ \\
\hline Duration from onset (years) & $9(5)$ & $12(4)$ & $10(3)$ & $9(3)$ & 0.012 \\
\hline Duration from diagnosis (years) & $5(3)$ & $8(3)$ & $6(2)$ & $5(4)$ & 0.001 \\
\hline \multicolumn{6}{|l|}{ Behavioural features } \\
\hline Disinhibition & 98 & 59 & 18 & 55 & - \\
\hline Apathy & 65 & 47 & 41 & 45 & - \\
\hline Loss of empathy & 33 & 28 & 18 & 14 & - \\
\hline Stereotyped behaviours & 95 & 72 & 24 & 28 & * \\
\hline Alt. food pref. & 58 & 50 & 41 & 23 & - \\
\hline Executive deficits & 59 & 25 & 6 & 0 & * \\
\hline \multicolumn{6}{|l|}{ Language features } \\
\hline Word finding & 45 & 84 & 71 & 100 & * \\
\hline Apraxia of speech & 5 & 6 & 94 & 32 & * \\
\hline Agrammatism & 0 & 0 & 53 & 14 & * \\
\hline Naming & 30 & 97 & 71 & 86 & - \\
\hline Single word comp & 5 & 84 & 18 & 18 & * \\
\hline Object agnosia & 2 & 34 & 0 & 5 & - \\
\hline Phonologic errors & 2 & 9 & 71 & 73 & * \\
\hline Word rep & 0 & 0 & 53 & 64 & * \\
\hline Sentence rep & 2 & 6 & 35 & 68 & * \\
\hline Sentence Comprehension & 23 & 72 & 88 & 68 & - \\
\hline Surface dyslexia & 11 & 81 & 71 & 64 & * \\
\hline \multicolumn{6}{|l|}{ Motor features } \\
\hline Parkinsonism & 18 & 3 & 6 & 9 & - \\
\hline Apraxia & 23 & 0 & 24 & 36 & - \\
\hline Gait disturbance & 12 & 3 & 12 & 9 & - \\
\hline Orobuccal apraxia & 8 & 3 & 24 & 0 & * \\
\hline \multicolumn{6}{|l|}{ Neuropsychiatric features } \\
\hline Delusions & 14 & 9 & 6 & 9 & - \\
\hline Hallucinations & 20 & 6 & 6 & 14 & - \\
\hline \multicolumn{6}{|l|}{ Other features } \\
\hline Prosopagnosia & 3 & 47 & 0 & 5 & * \\
\hline Rigidity of thought & 41 & 38 & 12 & 18 & - \\
\hline Irritability & 33 & 50 & 47 & 45 & - \\
\hline Aggression & 30 & 28 & 12 & 18 & - \\
\hline
\end{tabular}

*Features with high $(>80 \%)$ and low $(<20 \%)$ prevalences indicated and the patterns of features discriminating each clinical subtype statistically identified in bold $($ Wilks' lambda=0.024, $\mathrm{p}<0.0001)$.

bvFTD, behavioural variant frontotemporal dementia; Iv-PPA, logopenic variant-primary progressive aphasias; nfv-PPA, non-fluent/agrammatic variant-PPA; sv-PPA, semantic variant-PPA 
Table 2 Proportion of cases reclassified between FTD clinical phenotypes

\begin{tabular}{|c|c|c|c|c|}
\hline & $\begin{array}{l}\text { New } \\
\text { bvFTD } \\
(\mathrm{N}=66)\end{array}$ & $\begin{array}{l}\text { New } \\
\text { sv-PPA } \\
(\mathrm{N}=31)\end{array}$ & $\begin{array}{l}\text { New } \\
\text { nfv-PPA } \\
(\mathrm{N}=16)\end{array}$ & $\begin{array}{l}\text { New } \\
\text { Iv-PPA } \\
(\mathrm{N}=22)\end{array}$ \\
\hline Old bvFTD $(n=71)$ & 66 & 2 & 1 & 2 \\
\hline $\begin{array}{l}\text { Old semantic } \\
\text { dementia }(n=31)\end{array}$ & 0 & 27 & 0 & 2 \\
\hline $\begin{array}{l}\text { Old non-fluent } \\
\text { PPA }(n=33)\end{array}$ & 0 & 2 & 15 & 18 \\
\hline
\end{tabular}

bvFTD, behavioural variant frontotemporal dementia; Iv-PPA, logopenic variant-primary progressive aphasias; $n f v-P P A$, non-fluent/agrammatic variant-PPA sv-PPA, semantic variant-PPA.

language variants. The majority of cases previously diagnosed with semantic dementia were classified as sv-PPA (87\%, table 2). A small number $(6 \%)$ of cases previously diagnosed with semantic dementia had significant WFD, phonological errors and/or impaired repetition to be reclassified as lv-PPA. In addition, some previously classified bvFTD and nfv-PPA cases had sufficient sv-PPA specific deficits such as anomia and surface dyslexia to warrant reclassification into the new sv-PPA subtype.

The biggest change in FTD diagnoses was observed in previously diagnosed non-fluent PPA cases, where more than half (55\%) were reclassified as being lv-PPA (table 2). Most lv-PPA cases had a previous diagnosis of non-fluent PPA, with 9\% diagnosed previously as bvFTD and 9\% as sv-PPA. These cases had additional lv-PPA features warranting reclassification.

\section{New clinicopathological correlations}

Although the majority of clinically classified FTD cases had either FTLD-tau $(n=42 ; 31 \%)$ or FTLD-TDP $(n=48 ; 35 \%$, supplementary table e2), overall 33 patients (26\%) had AD pathology despite the fact that many met the new clinical criteria for one of the FTD variants not typically associated with AD (bvFTD, sv-PPA and nfv-PPA).

Patients meeting criteria for bvFTD mainly had FTLD-tau (45\%), FTLD-TDP (32\%) or FTLD-FUS (13\%), although all pathological subtypes were associated with bvFTD including AD $(10 \%)$ (figure 2A). Those with FTLD-FUS had significantly younger age at symptom onset $(\mathrm{p}<0.0001)$, diagnosis $(p<0.0001)$ and death $(p<0.0001)$ (see online supplementary table e2), in line with previously observed data. ${ }^{12}$ Analysis of clinical features that distinguish pathological subgroups of bvFTD revealed that executive dysfunction, parkinsonism and delusions were more common among cases with FTLD-TDP compared with FTLD-tau (see online supplementary table e3).

Cases with sv-PPA mainly had FTLD-TDP (68\%) with the remaining 10 patients having equal numbers of FTLD-tau and $\mathrm{AD}$ pathology (figure 2B). Phonological errors in speech appeared to be associated with underlying $\mathrm{AD}$ pathology $(p=0.047)$. In contrast, disinhibition was significantly associated with underlying FTLD pathology $(\mathrm{p}=0.016)$.

Most lv-PPA cases had AD (77\%, figure 2D) with the remainder split between FTLD-tau and FTLD-TDP. By contrast, 50\% of cases with nfv-PPA had FTLD-tau pathology, while a sizeable minority had $\operatorname{AD}(31 \%$, figure $2 \mathrm{C})$. No differences in the frequencies of clinical features across pathological groups were found in both nfv-PPA and lv-PPA clinical subgroups.

Discriminative analysis revealed that eight features discriminated those with FTLD from those with AD pathology with $86 \%$ accuracy (Wilks' lambda $=0.596, p<0.0001$, table 3 ). For
Table 3 Demographics and the proportion of cases in each group with the main clinical features of the new FTD clinical phenotypes

\begin{tabular}{|c|c|c|c|}
\hline & FTLD $(\mathrm{N}=102)$ & $A D(N=33)$ & $p$ Value \\
\hline$\%$ male $/ \%$ female & $64 / 36$ & $73 / 27$ & 0.211 \\
\hline Age at onset (years) & $58(9)$ & $64(10)$ & 0.002 \\
\hline Age at diagnosis (years) & $62(8)$ & $68(9)$ & 0.002 \\
\hline Age at death (years) & $68(9)$ & $74(8)$ & 0.003 \\
\hline Duration from onset (years) & $10(4)$ & $10(6)$ & 0.884 \\
\hline Duration from diagnosis (years) & $6(3)$ & $6(3)$ & 0.889 \\
\hline \multicolumn{4}{|l|}{ Behavioural features } \\
\hline Disinhibition & 79 & 51.5 & - \\
\hline Apathy & 58 & 36 & - \\
\hline Loss of empathy & 29 & 18 & - \\
\hline Stereotyped behaviours & 77 & 48 & - \\
\hline Alt. food pref. & 55 & 24 & * \\
\hline Executive deficits & 40 & 21 & - \\
\hline \multicolumn{4}{|l|}{ Language features } \\
\hline Word finding & 61 & 85 & * \\
\hline Apraxia of speech & 16 & 33 & - \\
\hline Agrammatism & 7 & 15 & - \\
\hline Naming & 56 & 73 & - \\
\hline Single word comp & 27 & 24 & - \\
\hline Object agnosia & 11 & 3 & * \\
\hline Phonologic errors & 12 & 58 & * \\
\hline Word rep & 8 & 42 & - \\
\hline Sentence rep & 10 & 42 & * \\
\hline Sentence Comprehension & 47 & 58 & - \\
\hline Surface dyslexia & 36 & 64 & - \\
\hline \multicolumn{4}{|l|}{ Motor features } \\
\hline Parkinsonism & 11 & 15 & - \\
\hline Apraxia & 17 & 30 & - \\
\hline Gait disturbance & 7 & 18 & * \\
\hline Orobuccal apraxia & 8 & 3 & - \\
\hline \multicolumn{4}{|l|}{ Neuropsychiatric features } \\
\hline Delusions & 8 & 18 & * \\
\hline Hallucinations & 11 & 21 & * \\
\hline \multicolumn{4}{|l|}{ Other features } \\
\hline Prosopagosia & 17 & 0 & - \\
\hline Rigidity of thought & 34 & 24 & - \\
\hline Irritability & 41 & 36 & - \\
\hline Aggression & 27 & 18 & - \\
\hline
\end{tabular}

${ }^{*}$ Features with high $(>80 \%)$ and low $(<20 \%)$ prevalences indicated and the patterns of features discriminating pathology type statistically identified in bold (Wilks' lambda $=0.596, \mathrm{p}<0.0001$ ).

AD, Alzheimer's disease; FTD, frontotemporal dementia; FTLD, frontotemporal lobar degeneration.

AD pathology, the discriminating features were a high prevalence of WFD, phonological errors, delusions and lack of object agnosia (table 3). FTLD pathology, on the other hand, was distinguished by the relative lack of neuropsychiatric features, phonological errors and gait disturbance. Based on these discriminating features, analysis of correct classification revealed that $93 \%$ of FTLD cases were predicted correctly, whereas it was only $64 \%$ for the $\mathrm{AD}$ cases. In other words, $36 \%$ of $\mathrm{AD}$ cases cannot be differentiated from those with FTLD pathologies based on current diagnostic clinical features.

\section{DISCUSSION}

This study assessed the new FTD clinical diagnostic criteria against the new pathological criteria for these syndromes and for AD. It has been well established that a proportion of cases 
with PPA have AD rather than FTLD pathology, and the new variant lv-PPA has been established in the hope of identifying these patients. ${ }^{2} 413$ The present study confirms that the new lv-PPA diagnosis mostly have pathological AD (77\%) and the distribution of FTLD pathologies across the other clinical syndromes was largely as previously reported. ${ }^{4} 5$ While the new clinical criteria were able to discriminate most cases of FTLD from $\mathrm{AD}$ presenting with lv-PPA, a proportion of all clinical FTD phenotypes had pathological AD. These included $\sim 10 \%$ of bvFTD, $\sim 15 \%$ of sv-PPA and $\sim 30 \%$ of nfv-PPA. In terms of the clinical FTD syndromes associated with AD pathology, only $\sim 50 \%$ had lv-PPA with nearly equal numbers of bvFTD, sv-PPA and nfv-PPA making up the other $50 \%$ of AD cases. Further analyses were performed to determine distinguishing and discriminating features between each FTD syndrome as well as between those with underlying FTLD and AD pathology.

Our study largely validates the new FTD clinical diagnostic criteria in a large group of FTD cases comprising approximately a half behavioural variant and a half language variants, reflecting frequencies documented in other large series. ${ }^{14} 15$ There have been a number of recent studies comparing the new versus the previous FTD diagnostic criteria; however, few have autopsy confirmation. A clinical comparison between the new versus previous criteria for bvFTD ${ }^{16}$ found that $33 \%$ more cases would be currently diagnosed using the new criteria, in particular those previously excluded with spatial disorientation and early severe amnesia. As we and others ${ }^{17}$ have shown that a proportion of patients with previously diagnosed bvFTD have AD at autopsy, it will be important to determine whether such a large number of newly diagnosed bvFTD cases will have non-FTD pathologies over time. A similar clinical comparison of the new versus previous criteria for PPA syndromes suggests that $\sim 40 \%$ of the 46 patients assessed could not be classified into any single new category as most met multiple criteria. ${ }^{18}$ In the present study, we were able to identify an initial dominant symptom in the majority of cases, although around $30 \%$ of cases had multiple diagnostic features (see online supplementary material) and required consensus conferencing to determine their final diagnosis. We therefore analysed the most discriminating features for each of the clinical syndromes.

Only a small proportion of the features used in the criteria assisted with clinical discrimination. Apraxia of speech and agrammatism differentiated between nfv-PPA and lv-PPA, while executive deficits differentiated bvFTD and sv-PPA from $n f v-P P A$ and lv-PPA. Orobuccal apraxia was highly specific for the nfv-PPA variant, perhaps unsurprising as it often co-occurs with apraxia of speech (impaired coordination of speech resulting in groping, pauses and loss of prosody), ${ }^{19} 20$ a core diagnostic feature of nfv-PPA. A core set of 12 features (stereotypic behaviour, executive dysfunction, WFD, AoS, agrammatism, SWC, phonologic errors, word and sentence repetition, surface dyslexia, orobuccal apraxia and prosopagnosia, table 1) assisted with discrimination between clinical FTD syndromes, with a further five features (altered food preferences, object agnosia, gait disturbance, hallucinations and delusions, table 3) assisting with further discrimination of cases with AD pathology. WFD, phonological errors, gait disturbance and neuropsychiatric features were found to be more prevalent in those with $\mathrm{AD}$ pathology, whereas object agnosia differentiated those with FTLD pathology. Language dysfunction in AD is well established, and the syndrome of lv-PPA was proposed to account for the majority of cases with atypical AD presenting with aphasia, the core features of which are anomia with impaired span. ${ }^{21-23}$ In keeping with our finding, recent studies have suggested phonological errors rather than anomia or poor span may be a more sensitive predictor of $\mathrm{AD}$ as indexed by Pittsburgh Compound B (PiB) uptake. ${ }^{24}$ Patients with lv-PPA have atrophy involving the angular and superior temporal gyri known to be critical for phonological processing. ${ }^{25} 26$

Importantly, a small number of cases changed classification when the new criteria were applied. They were predominantly cases previously labelled non-fluent progressive aphasia, reclassified as lv-PPA, which is a relatively new syndrome compared with the other variants of PPA. ${ }^{27}$ Although the defining features of the PPA variants, particularly the separation of nfv-PPA from lv-PPA, remain somewhat controversial, ${ }^{18} 2829$ studies using PiB as an $\mathrm{AD}$ biomarker have generally supported the tripartite classification. Despite the retrospective nature of the present study, we were able to correctly identify cases with lv-PPA who largely exhibited $\mathrm{AD}$ pathology, providing a good evidence base for continuing to separate such cases from the other PPA types.

Most surprising was the finding that a third of cases with $\mathrm{AD}$ pathology could not be differentiated from those with clinical FTD syndromes based on current diagnostic features, in that they presented with bvFTD, sv-PPA or nfv-PPA. This finding is supported by other studies involving small cohorts. ${ }^{30} 31$ A proportion of patients with AD pathology will be clinically misdiagnosed as having an FTD syndrome, even after excluding those with lv-PPA. Particular features of these misdiagnosed cases were prominent neuropsychiatric features (delusions and hallucinations), as suggested by others, ${ }^{30}$ and/or specific language features (WFD, phonological errors, repetition deficits). It is possible that a proportion of the nfv-PPA would be classified as lv-PPA if assessed prospectively as the criteria for diagnosis are complex to apply retrospectively and key differentiating features may have been overlooked, but this an unlikely explanation in all cases and would not account for the cases with sv-PPA or bvFTD and AD pathology. In keeping with our finding that features indicating executive dysfunction fail to discriminate $\mathrm{AD}$ from bvFTD, a recent study found poor separation on standard tests of attention and working memory compared with tests based on social cognition and antisaccadic accuracy. ${ }^{31}$ There is clearly a small group of $\mathrm{AD}$ patients with disproportionate frontal pathology who are difficult to identify since they met the current clinical criteria for bvFTD. Of interest, such cases rarely had altered food preferences, which may prove therefore to be a good indicator of FTLD pathology. ${ }^{32}$

The prevalence of AD pathology in clinical FTD reported in this series perhaps prompts the consideration of 'hallmark' features of $\mathrm{AD}$ such as episodic memory to be included in further discriminatory analyses. Indeed, the overlap of such features in FTD and AD has been reported leading to cognitive and imaging studies that have shown distinct and differing profiles of episodic memory impairment between FTD variants and $\mathrm{AD} .{ }^{33} 34$ However, it is evident that neuropathological diagnoses would be a valuable addition to such analyses and could potentially assist in finding more robust clinical features that dissociate FTD patients with AD pathology from those with FTLD.

The potential limitations of this study include the retrospective assessment of the clinical features, as missing data for features were interpreted as negative results. In addition, the behavioural features were mainly informant-derived with the inherent difficulty of the subjective nature of such information. Of course, there are limited autopsies on phenocopies and/or alternate pathologies, and these are not represented in the present series. Genetics and family history of disease has only risen to prominence subsequent to the majority of these cases coming to autopsy and are not currently formalised into 
diagnostic criteria, but will be important to assess for their impact in future studies. There is a need to determine 'early' features that can aid in the correct diagnosis to guide appropriate therapies. Our assessment would indicate there would be fewer discriminatory clinical features if only early features were included in the analyses, but their further identification and development into a core battery together with information from imaging and genotyping will be an important next step.

Acknowledgements This study was only possible because of the generous donation by participating patients and families. We wish to thank the staff of the Sydney Brain Bank for tissue preparation and initial characterisation of the Sydney cases (supported by the NHMRC (enabling grants 282933 and 605210), the University of New South Wales and Neuroscience Research Australia), the staff of the Cambridge Brain Bank for tissue preparation of the Cambridge cases and Dr John H Xuereb for initial neuropathological diagnosis of the Cambridge cases. We would also like to thank Heidi Cartwright for the figures.

Contributors LC assisted with the final design and conceptualisation of the study, clinical data collection, analysis and interpretation of the data, and drafting the manuscript. JRH initially designed and conceptualised the study, clinician responsible for specialist clinics and longitudinal brain donor programme for FTD syndromes, clinical and pathological case ascertainment, oversight of clinical data collection, interpretation of the data, and revised the manuscript. CEL was responsible for clinical case ascertainment, oversight of clinical data collection, analysis and interpretation of the data, and revised the manuscript. CMcG and RHT were responsible for pathological data collection, interpretation of pathological data, and revised the manuscript. JJK initially designed and conceptualised the study, clinical and pathological case ascertainment, oversight of pathological data collection, analysis and interpretation of the data, and drafting the manuscript. GMH designed and conceptualised the study, was responsible for longitudinal brain donor programme in Sydney, clinical and pathological case ascertainment, oversight of clinical and pathological data collection, analysis and interpretation of the data, and drafting the manuscript.

Funding Australian Government Postgraduate Award to LC; Neuroscience Research Australia Postgraduate top-up to LC; National Health and Medical Research Council of Australia (NHMRC) (project grants 1025065 to $\mathrm{JK}$ and JH and 1029538 to GH, $\mathrm{JH}$ and JK, programme grant 1037746 to $\mathrm{GH}$, JK and $\mathrm{JH}$, senior principal research fellowship to GH); autopsy collection through the Cambridge Brain Bank was supported by the UK Medical Research Council; autopsy collection through the Sydney Brain Bank was supported by the NHMRC (enabling grants 282933 and 605210), the University of New South Wales and Neuroscience Research Australia.

Competing interests None.

Patient consent Obtained.

Ethics approval Addenbrooke's Hospital Local Ethics Committee and the Human Ethics Committees of the South Eastern and Illawarra Area Heath Service and the Universities of New South Wales and Sydney.

Provenance and peer review Not commissioned; externally peer reviewed.

Data sharing statement Data from this study are available to those listed on the ethics review information, as the clinical records are not de-identified.

\section{REFERENCES}

1 Rascovsky K, Hodges JR, Knopman D, et al. Sensitivity of revised diagnostic criteria for the behavioural variant of frontotemporal dementia. Brain 2011;134:2456-77.

2 Gorno-Tempini ML, Hillis AE, Weintraub $\mathrm{S}$, et al. Classification of primary progressive aphasia and its variants. Neurology 2011;76:1006-14.

3 Mackenzie IR, Neumann M, Bigio EH, et al. Nomenclature and nosology for neuropathologic subtypes of frontotemporal lobar degeneration: an update. Acta Neuropathol 2010;119:1-4.

4 Mesulam M, Wicklund A, Johnson N, et al. Alzheimer and frontotemporal pathology in subsets of primary progressive aphasia. Ann Neurol 2008;63:709-19.

5 Deramecourt $\mathrm{V}$, Lebert $\mathrm{F}$, Debachy $\mathrm{B}$, et al. Prediction of pathology in primary progressive language and speech disorders. Neurology 2010;74:42-9.

6 McKhann GM, Albert MS, Grossman M, et al. Clinical and pathological diagnosis of frontotemporal dementia: report of the Work Group on Frontotemporal Dementia and Pick's Disease. Arch Neurol 2001;58:1803-9.
7 Neary D, Snowden JS, Gustafson L, et al. Frontotemporal lobar degeneration: a consensus on clinical diagnostic criteria. Neurology 1998;51:1546-54.

8 Cairns NJ, Bigio EH, Mackenzie IR, et al. Neuropathologic diagnostic and nosologic criteria for frontotemporal lobar degeneration: consensus of the Consortium for Frontotemporal Lobar Degeneration. Acta Neuropathol 2007;114:5-22.

9 Consensus recommendations for the postmortem diagnosis of Alzheimer's disease. The National Institute on Aging, and Reagan Institute Working Group on Diagnostic Criteria for the Neuropathological Assessment of Alzheimer's Disease. Neurobiol Aging 1997;18:S1-2.

10 Hyman BT, Phelps CH, Beach TG, et al. National Institute on Aging-Alzheimer's Association guidelines for the neuropathologic assessment of Alzheimer's disease. Alzheimers Dement 2012;8:1-13.

11 Tan RH, Shepherd CE, Kril JJ, et al. Classification of FTLD-TDP cases into pathological subtypes using antibodies against phosphorylated and non-phosphorylated TDP43. Acta Neuropathol Commun 2013;1:33.

12 Urwin H, Josephs KA, Rohrer JD, et al. FUS pathology defines the majority of tauand TDP-43-negative frontotemporal lobar degeneration. Acta Neuropathol 2010;120:33-41

13 Leyton CE, Villemagne VL, Savage S, et al. Subtypes of progressive aphasia: application of the International Consensus Criteria and validation using beta-amyloid imaging. Brain 2011;134:3030-43.

14 Kertesz $A$, McMonagle $P$, Blair $M$, et al. The evolution and pathology of frontotemporal dementia. Brain 2005;128:1996-2005.

15 Shi J, Shaw CL, Du Plessis D, et al. Histopathological changes underlying frontotemporal lobar degeneration with clinicopathological correlation. Acta Neuropathol 2005;110:501-12

16 Costa S, Suarez-Calvet M, Anton S, et al. Comparison of 2 diagnostic criteria for the behavioral variant of frontotemporal dementia. Am J Alzheimers Dis Other Demen 2013;28:469-76.

17 Harris JM, Gall C, Thompson JC, et al. Sensitivity and specificity of FTDC criteria for behavioral variant frontotemporal dementia. Neurology 2013;80:1881-7.

18 Sajjadi SA, Patterson K, Arnold RJ, et al. Primary progressive aphasia: a tale of two syndromes and the rest. Neurology 2012;78:1670-7.

19 Ogar JM, Dronkers NF, Brambati SM, et al. Progressive nonfluent aphasia and its characteristic motor speech deficits. Alzheimer Dis Assoc Disord 2007;21: S23-30.

20 Rohrer JD, Rossor MN, Warren JD. Apraxia in progressive nonfluent aphasia. J Neurol 2010;257:569-74.

21 Kirshner HS, Webb WG, Kelly MP. The naming disorder of dementia. Neuropsychologia 1984;22:23-30.

22 Robinson KM, Grossman M, White-Devine T, et al. Category-specific difficulty naming with verbs in Alzheimer's disease. Neurology 1996;47:178-82.

23 Murdoch BE, Chenery HJ, Wilks V, et al. Language disorders in dementia of the Alzheimer type. Brain Lang 1987;31:122-37.

24 Croot K, Ballard K, Leyton CE, et al. Apraxia of speech and phonological errors in the diagnosis of nonfluent/agrammatic and logopenic variants of primary progressive aphasia. J Speech Lang Hear Res 2012;55:S1562-72.

25 Rohrer JD, Ridgway GR, Crutch SJ, et al. Progressive logopenic/phonological aphasia: erosion of the language network. Neuroimage 2010;49: 984-93.

26 Leyton $C E$, Piguet 0 , Savage $S$, et al. The neural basis of logopenic progressive aphasia. J Alzheimers Dis 2012;32:1051-9.

27 Gorno-Tempini ML, Dronkers NF, Rankin KP, et al. Cognition and anatomy in three variants of primary progressive aphasia. Ann Neurol 2004;55:335-46.

28 Ash S, McMillan C, Gunawardena D, et al. Speech errors in progressive non-fluent aphasia. Brain Lang 2010;113:13-20.

29 Thompson CK, Cho S, Hsu CJ, et al. Dissociations Between Fluency And Agrammatism In Primary Progressive Aphasia. Aphasiology 2012;26:20-43.

30 Mendez MF, Joshi A, Tassniyom K, et al. Clinicopathologic differences among patients with behavioral variant frontotemporal dementia. Neurology 2013:80:561-8

31 Possin KL, Feigenbaum D, Rankin KP, et al. Dissociable executive functions in behavioral variant frontotemporal and Alzheimer dementias. Neurology 2013;80:2180-5.

32 Piguet $\mathrm{O}$, Petersen A, Yin Ka Lam B, et al. Eating and hypothalamus changes in behavioral-variant frontotemporal dementia. Ann Neurol 2011;69:312-19.

33 Graham KS, Hodges JR. Differentiating the roles of the hippocampal complex and the neocortex in long-term memory storage: evidence from the study of semantic dementia and Alzheimer's disease. Neuropsychology 1997;11:77-89.

34 Pennington C, Hodges JR, Hornberger M. Neural correlates of episodic memory in behavioral variant frontotemporal dementia. J Alzheimers Dis 2011;24:261-8. 